Rev. Int. Contam. Ambie. 33 (Especial Biotecnología e ingeniería ambiental) 63-72, 2017

DOI: 10.20937/RICA.2017.33.esp01.06

\title{
EVALUACIÓN DE LA BIODEGRADACIÓN DE ATRAZINA PURA Y COMERCIAL (GESAPRIM) EN SUELOS ARENOSOS, LIMOSOS Y ARCILLOSOS
}

\author{
Rosa Angélica GUILLÉN GARCÉS*, Cristina LIZAMA BAHENA, Ana Gabriela ORTEGA HERNÁN- \\ DEZ, Gabriela Eleonora MOELLER CHÁVEZ y Luis Gerardo TREVIÑO QUINTANILLA
}

Departamento de Tecnología Ambiental, Universidad Politécnica del estado de Morelos. Bulevar Cuauhnáhuac 566, Colonia Lomas del Texcal, Jiutepec, Morelos, México, C. P. 62550

*Autor para correspondencia: aguillen@upemor.edu.mx

(Recibido julio 2015; aceptado noviembre 2016)

Palabras clave: herbicida, disponibilidad, vida media

\section{RESUMEN}

La atrazina es ampliamente utilizada en México y el estado de Morelos en la eliminación de maleza de hoja ancha en cultivos de maíz, sorgo, caña de azúcar y piña. Su uso está prohibido en la Unión Europea y restringido en Estados Unidos de América. Hasta el momento sólo se han caracterizado los procesos que influyen en la migración de atrazina sin presencia de emulsificantes y surfactantes. El presente trabajo tiene como objetivo caracterizar la adsorción y la biodegradación de atrazina al $100 \%$ y gesaprim (producto comercial con $43 \%$ atrazina y mezclado con surfactantes y emulsificantes), en diferentes muestras de suelo agrícola del estado de Morelos. Se hicieron muestreos en tres municipios: Moyotepec, Yecapixtla, y Huautla, con base en las diferentes texturas del suelo (arenosos, limosos y arcillosos). Los suelos fueron caracterizados fisica y químicamente, asimismo se obtuvieron las cinéticas de biodegradación con atrazina al $100 \%$ como ingrediente activo y con el producto comercial gesaprim que contiene $43 \%$ de atrazina como ingrediente activo.. Las vidas medias en los suelos Moyotepec, Yecapixtla y Huautla para el gesaprim fueron 126, 18 y $40 \mathrm{~d}$ y los porcentajes de biodegradación de $21.4,71.8$ y $50.1 \%$. Para atrazina al $100 \%$, fueron de 17,16 y 6 d y los porcentajes de degradación de $52.7,72.9$ y $65.8 \%$, respectivamente. Con base en las vidas medias de atrazina al $100 \%$ y gesaprim (43\% atrazina), se observó que la presencia de surfactantes y humectantes disminuye la velocidad de biodegradación y el porcentaje de biodegradación, con lo que se favorecen otros procesos de disipación, debido probablemente a que aumenta la disponibilidad del herbicida.

Key words: herbicide, availability, half-life

\begin{abstract}
Atrazine is widely used in Mexico and in the state of Morelos to eliminate broadleaf weeds in maize, sorghum, sugarcane and pineapple crops. Its use is prohibited in the European Union and restricted in the United States of America. So far, only processes that influence atrazine migration without the presence of emulsifiers and surfactants have been characterized. The present work aims to characterize the adsorption and biodegradation of pure atrazine (100\%) and gesaprim (commercial product with $43 \%$ atrazine mixed with surfactants and emulsifiers), in different samples of agricultural
\end{abstract}


soils of the state of Morelos. Samplings were taken in three municipalities: Moyotepec, Yecapixtla and Huautla, based on the difference in soil texture (sand, silt and clay). The soils were physicochemically characterized and biodegradation kinetics was obtained in the different soil samples, using a similar field recommended concentration. Atrazine was applied as $100 \%$ active ingredient and gesaprim commercial product containing 43 $\%$ of atrazine as active ingredient. Half-lives in soils from Moyotepec, Yecapixtla and Huautla for gesaprim were 126, 18 and $40 \mathrm{~d}$ with biodegradation percentages of 21.4, 71.8 and $50.1 \%$. For atrazine (100\%), the half-life was 17,16 and $6 \mathrm{~d}$ with degradation percentages of $52.7,72.9$ and $65.8 \%$, respectively. Based on the half-lives of atrazine $100 \%$ and gesaprim ( $43 \%$ atrazine), it was observed that the presence of surfactants and humectants decreases the rate and percentage of biodegradation, favoring other dissipation processes, probably due to the increase in availability of the herbicide.

\section{INTRODUCCIÓN}

La atrazina (6-Cloro- $\mathrm{N}^{2}$-etil- $\mathrm{N}^{4}$-isopropil-1,3,5triazina-2,4-diamina) con número CAS: 1912-24-9 es un herbicida triazínico selectivo pre y post emergente ampliamente usado para la eliminación de maleza de hoja ancha en cultivos de sorgo, caña de azúcar, trigo, soya, frijol, plátano y piña, entre otros. A nivel mundial su uso se inició en 1958 y se ha incrementado en los últimos 50 años (Golla et al. 2011). En México específicamente, comenzó a utilizarse en 1975 y actualmente se sigue empleando (SAGARPA 2007). Estudios en ranas han determinado que es un inductor de hermafrodismo en concentraciones tan bajas como $0.1 \mu \mathrm{g} / \mathrm{L}$ (Hayes et al. 2002). La exposición de los seres humanos a la atrazina se ha relacionado con efectos agudos, tales como irritación de ojos, piel, nariz y garganta. Efectos crónicos, como alteraciones en las funciones del corazón, hígado y riñón, así como problemas de reproducción y modificaciones en los niveles de hormonas que afectan la ovulación, provocando malformaciones. Asimismo se considera un posible cancerígeno (ATSDR 2003, Rusiecki 2004). Debido a sus características fisicas y químicas, movilidad y persistencia en el ambiente se ha considerado un contaminante orgánico persistente, aunque su clasificación e integración al anexo de Estocolmo está en revisión. Es un compuesto poco soluble en agua y posee una alta movilidad en el suelo debido a que se aplica mezclado con surfactantes y humectantes, lo que aumenta el riesgo de contaminación de los cuerpos de agua superficiales y subterráneos por infiltración y escorrentía.

Entre los procesos que intervienen en la atenuación natural de la atrazina, la biodegradación es el principal mecanismos de disipación en el ambiente (Ma y Selim 1996) y es afectado por factores como el tipo de suelo, el contenido de materia orgánica (Ling et al. 2005) y el pH (Tyess et al. 2006). Considerando que existen diversos factores que afectan la biode- gradación de atrazina se ha encontrado un intervalo amplio entre las vidas medias de este compuesto, con valores que van desde 0.99 hasta $5824 \mathrm{~d}$ (Schoen y Winterlin 1987, Guillén-Garcés et al. 2007).

En el caso del tipo de textura de suelo, se ha encontrado que en los arenosos, la disponibilidad de contaminantes aumenta en comparación con los arcillosos, debido principalmente a que las arcillas tienen mayor capacidad de adsorción que las arenas, por lo que las primeras pueden limitar los procesos de biodegradación. Esta limitación en la biodegradación puede deberse a que los compuestos no se encuentran disponibles para ser degradados por los microorganismos con capacidad de biodegradar el herbicida (Huang et al. 2003). Respecto a la materia orgánica se ha encontrado que suelos con alto contenido de ésta puede limitar la biodegradación de atrazina, ya que el herbicida tiende a adsorberse en esta fracción de suelo (González-Márquez y Hansen 2009). Aunque esta limitación también dependerá de la presencia de microorganismos capaces de degradarla, dado que se ha demostrado que la aplicación frecuente del herbicida en el suelo tiende a modificar la población microbiana presente (Ross et al. 2006). Entre estas modificaciones están la inducción de enzimas en los microorganismos, debido a la adaptación de las comunidades microbianas a la presencia de atrazina, con lo que se favorece la biodegradación del herbicida en el suelo (Mahía y Díaz-Raviña 2007). Otro factor que influye sobre la biodegradación de la atrazina es el $\mathrm{pH}$ del suelo, al ser un compuesto que presenta característica de base débil, éste se oxida a $\mathrm{pH}$ por debajo de 4 , por lo que tiende a adsorberse en suelos con $\mathrm{pH}$ bajos (Wang et al. 2011). La adsorción por hidrofobicidad en la fracción orgánica del suelo es importante, tal vez más que la retención por intercambio iónico ya que el pH promedio en los suelos va de 6 a 8 .

Por lo anterior, es un compuesto que se ha caracterizado como móvil, encontrándose en diversos cuerpos 
de agua, tanto superficiales como subterráneos (Farré et al. 2007). El límite máximo permisible en agua para consumo humano establecido por la Organización Mundial de la Salud (OMS) es $2 \mathrm{mg} / \mathrm{L}$. Además, se ha prohibido su uso en la Unión Europea y restringido en países como Estados Unidos y Australia (Beth y Colangelo 2006). Por otro lado, en Asia, Canadá, América Latina, el Caribe y México su uso está permitido (UNEP 2002, FAO 2005, PMRA 2015).

En México, es el tercer herbicida más utilizado, se ha reportado que sólo en los distritos de riego del país se utilizan 1078 t/año (Villada-Canela 2006) y se tiene registrado su uso ante la Comisión Intersecretarial para el Control del Proceso y Uso de Plaguicidas y Sustancias Tóxicas (CICOPLAFEST 2004), pero no existe una regulación de su aplicación. Su principal nombre comercial es gesaprim, el cual contiene desde 43 a $90 \%$ de ingrediente activo, según su presentación como suspensión o granular, respectivamente, además de aditivos como humectantes y surfactantes, con las que se mezclan para aumentar la solubilidad del compuesto. Singh et al. (2014), demostraron que la adición de humectantes y surfactantes afecta la biodegradación de atrazina. Encontraron que la presencia de estos aditivos favorece la biodegradación de atrazina en suelos contaminados debido a que incrementan la solubilidad, además se promueven la desorción e incrementan la biodisponibilidad de la atrazina en suelo, mientras que la presencia de aditivos sintéticos limita la biodegradación de este herbicida.

El estado de Morelos por su edafología está dividido en zonas con suelos arenosos, limosos y arcillosos. En cada una de estas zonas se encuentran productores de cultivos donde se aplica gesaprim. Hasta la fecha no se han caracterizado los procesos de biodegradación y adsorción de este compuesto puro y comercial en suelos agrícolas con diferentes texturas. El objetivo del presente trabajo fue caracterizar la biodegradación y adsorción de atrazina, como ingrediente activo al $100 \%$ y presente en gesaprim (43\% atrazina), en suelos arenosos, limosos y arcillosos de uso agrícola del estado de Morelos.

\section{MATERIALES Y MÉTODOS}

Se seleccionaron tres zonas del estado de Morelos con diferentes tipos de suelo (Fig. 1). La primera se ubica en el municipio de Moyotepec y está clasificada como un suelo de tipo Phaeozem (INEGI 2005). El clima es templado y húmedo con vegetación natural de pastos altos o bosques. Tiene suelos oscuros y ricos en materia orgánica que actualmente se utilizan para la producción de soya, trigo, cebada y hortalizas, con una conductividad hidráulica de $1.3 \mathrm{~cm} / \mathrm{h}$ (FAO 2003). El segundo sitio de muestreo se ubica en el municipio de Yecapixtla, el tipo de suelo es Vertisol (INEGI 2005) y se caracteriza por su alto contenido de arcillas que se expanden con la humedad y se contraen con la sequía. Sus colores más comunes son el negro o gris oscuro. El clima en la zona es semiárido a subhúmedo y de tipo mediterráneo. Se utilizan para la producción de caña, cereales, hortalizas y algodón. Tienen baja susceptibilidad a la erosión y alto riesgo de salinización, con una conductividad hidráulica de $0.05 \mathrm{~cm} / \mathrm{h}$ (FAO 2003). El tercer sitio de muestreo se encuentra situado en Huautla, presenta un tipo de suelo Leptosol (INEGI 2005), el cual es suelo poco profundo, pedregoso y poco desarrollado que puede contener una gran cantidad de material calcáreo, además tiene una capa superficial rica en materia orgánica que le confiere un mayor potencial de aprovechamiento agrícola. La zona es poco permeable debido a la presencia de derrames basálticos, presenta climas cálidos del sur y centro y templados y semifríos del norte del estado.En la zona se cultiva principalmente maíz, fríjol, chile, cacahuate y calabaza (SEDESU 2013), la conductividad hidráulica que presenta es de $5 \mathrm{~cm} / \mathrm{h}$ (FAO 2003).

El método de muestreo fue de tipo superficial considerando sólo la capa arable de $30 \mathrm{~cm}$ de profundidad. De los terrenos se tomaron 21 muestras simples y se mezclaron para obtener una muestra compuesta. La muestra compuesta se tamizó a través de una malla No. 6 correspondiente a $4.76 \mathrm{~mm}$ de apertura para homogenizarla. Los suelos fueron caracterizados fisica y químicamente respecto a $\mathrm{pH}$, humedad, materia orgánica, capacidad de campo, densidad aparente y textura(ASTM 1987, 1995, 1998 y 2011). Una vez que se caracterizaron los suelos, se realizaron isotermas de adsorción con base en la guía TG 106 de la Organización para la Cooperación y el Desarrollo Económico (OCDE 2000). El procedimiento fue colocar en tubos de teflón $2 \mathrm{~g}$ de suelo seco y agregarle $10 \mathrm{~mL} \mathrm{CaCl} 2$ $0.01 \mathrm{M}$, se dejaron reposar durante $24 \mathrm{~h} \mathrm{a} 60{ }^{\circ} \mathrm{C}$, lo anterior para limitar en los suelos el proceso de biodegradación mientras se alcanzaba el equilibrio entre la atrazina disuelta y adsorbida. Después se agregó a una serie de tubos solución de atrazina al $100 \%$ y a otra serie gesaprim ( $43 \%$ atrazina) con $\mathrm{CaCl}_{2}$, hasta alcanzar concentraciones de atrazina de $0,80,120$ y $240 \mathrm{mg} / \mathrm{L}$. Posteriormente se pusieron en agitación a $80 \mathrm{rpm}$ durante $24 \mathrm{~h}$ y se centrifugaron las mezclas a 6000 rpm para obtener los extractos y determinar la cantidad de atrazina disuelta. Para las cinéticas de biodegradación se pesó por triplicado $250 \mathrm{~g}$ de suelo 


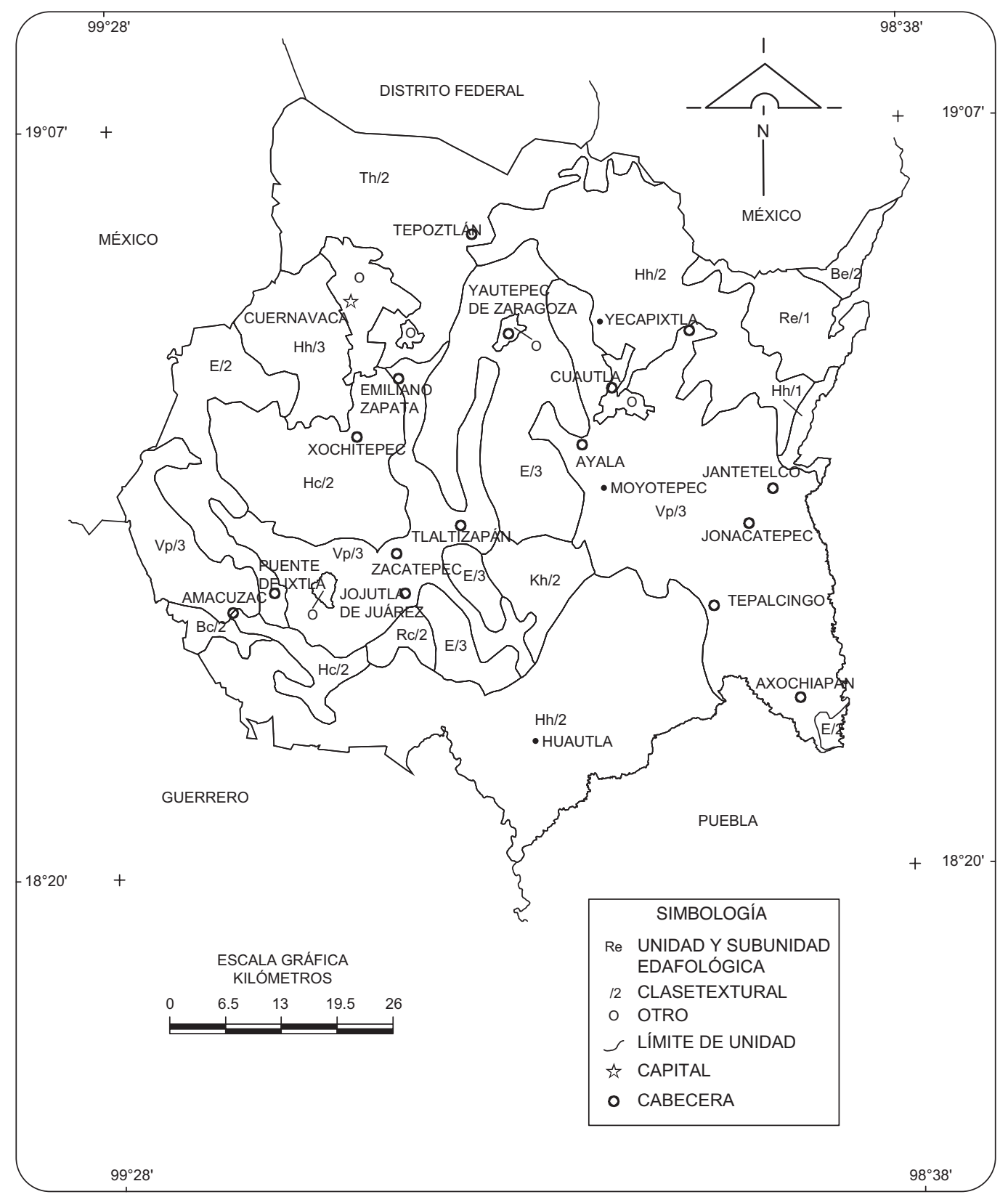

Fig. 1. Zonas de muestreo seleccionadas en el estado de Morelos con base al tipo de suelo INEGI (2005)

y se aplicó atrazina en concentración similar a la dosis recomendada por los fabricantes para cultivos de maíz de $3.7 \mathrm{mg} / \mathrm{kg}$, que equivale a $2 \mathrm{~kg} / \mathrm{ha}$. La aplicación se realizó en dos presentaciones, la primera como ingrediente activo al $100 \%$ y la segunda como producto comercial gesaprim (43\% atrazina). Se realizaron las correcciones pertinentes para que en todos los experimentos de biodegradación la concentración de atrazina fuera la misma. Las condiciones experimentales fueron $50 \%$ de la capacidad de campo y se incubaron a $32 \pm 1{ }^{\circ} \mathrm{C}$ durante 29 d. A diferentes tiempos se tomó una muestra de $25 \mathrm{~g}$ de suelo y se le adicionó en proporción 1:1 masa:volumen una mezcla de solventes compuesta por acetona: metanol: ácido acético en una proporción de 63:25:12 v/v para la extracción del ingrediente activo y se colocó en agitación durante $17 \mathrm{~h}$ a $120 \mathrm{rpm}$. Las muestras se centrifugaron a $6000 \mathrm{rpm}$ para obtener el sobrenadante, el cual fue evaporado hasta $1 \mathrm{~mL}$ en un rotaevaporador a una temperatura de 70 a $75^{\circ} \mathrm{C}$ a $150 \mathrm{rpm}$. Posteriormente la atrazina contenida en el sobrenadante se extrajo con 
diclorometano, el cual se evaporó hasta alcanzar $1 \mathrm{~mL}$. Tras esta extracción se les adicionó $30 \mathrm{~mL}$ de metanol y se evaporó hasta sequedad, por último los extractos obtenidos se disolvieron en $10 \mathrm{~mL}$ de la mezcla de acetona: metanol y ácido acético. El porcentaje de recuperación de este método de extracción es del $82 \%$, mismo que fue considerado para las correcciones de concentración determinadas (Hansen et al. 2001).

De los extractos obtenidos en los experimentos de adsorción y biodegradación se inyectaron $40 \mu \mathrm{L}$ al equipo de cromatografía de líquidos de alta resolución (HPLC, por sus siglas en inglés) Perkin Elmer serie 200. La cuantificación de atrazina se realizó con una curva estándar que se inyectó en el cromatógrafo utilizando una columna $\mathrm{C}-18$ con un tamaño de partícula de $5 \mu \mathrm{m}$ y dimensiones de $4.5 \times 250 \mathrm{~mm}$, a través de un detector de UV-VIS a $220 \mathrm{~nm}$; flujo de $1 \mathrm{~mL} / \mathrm{min}$. La composición de las fases utilizadas fue (A) fosfato dibásico de potasio $\left(\mathrm{K}_{2} \mathrm{HPO}_{4}\right) 20 \mathrm{mM}$, pH 7 y (B) acetonitrilo, (Hansen et al. 2001), los gradientes de solventes se detallan en el cuadro I.

CUADRO I. GRADIENTES DE FASES PARA DETERMINACIÓN DE ATRAZINA EN LOS EXTRACTOS DE LOS SUELOS MEDIANTE CROMATOGRAFÍA DE LÍQUIDOS DE ALTA RESOLUCIÓN

\begin{tabular}{ccc}
\hline Tiempo (min) & A (\%) & B (\%) \\
\hline 0 & 80 & 20 \\
10 & 70 & 30 \\
12 & 70 & 30 \\
18 & 50 & 50 \\
\hline
\end{tabular}

$\mathrm{A}=$ Fosfato dibásico de potasio $20 \mathrm{mM}$

$\mathrm{B}=$ Acetonitrilo

La determinación de los parámetros cinéticos obtenidos en los experimentos de biodegradación se calcularon al ajustar la fase exponencial a una cinética de primer orden (ecuación 1), modelo matemático desarrollado por Hamaker en 1972.

$\ln \left[\frac{C a}{C a_{0}}\right]=k t$

Donde

$C a=$ Concentración de sustrato en un tiempo determinado $(\%)$

$C a_{0}=$ Concentración inicial de sustrato (\%)

$t=$ Tiempo (s)

$k=$ Constante de biodegradación $\left(\mathrm{d}^{-1}\right)$

A partir de la ecuación 1 se calculó la vida media $\left(t_{1 / 2}\right)$ del herbicida en suelo, así se modificó y se obtuvo la siguiente ecuación. $t_{1 / 2}=\frac{\ln 2}{k}$

\section{RESULTADOS Y DISCUSIONES}

Los análisis de $\mathrm{pH}$ indican que los suelos de Yecapixtla y Huautla fueron ligeramente ácidos, mientras que el de Moyotepec fue neutro (Cuadro II). Considerado que la atrazina es una base débil que se oxida a un $\mathrm{pH}$ menor a 4 , se puede favorecer su adsorción en sustancias húmicas a pH ácidos (Ahmad y Rahman 2009). También se ha reportado que el proceso de adsorción es independiente del $\mathrm{pH}$ para valores superiores a 4 (Weber 1995). Koskinen y Clay (1997) establecen esta independencia para valores superiores a 6 . Con base en ello, se puede decir que en los suelos estudiados no había influencia del pH sobre la adsorción de atrazina.

CUADRO II. CARACTERIZACIÓN FISICOQUÍMICA DE LAS MUESTRAS DE SUELO PROCEDENTES DEL ESTADO DE MORELOS.

\begin{tabular}{lccc}
\hline Parámetro & \multicolumn{3}{c}{ Muestra de suelo procedentes de: } \\
\cline { 2 - 4 } & Moyotepec & Yecapixtla & Huautla \\
\hline $\mathrm{pH}^{1}$ & 7.55 & 6.48 & 6.20 \\
Humedad (\%)peso/peso $^{2}$ & 15.03 & 19.50 & 15.90 \\
Materia orgánica (\%) $^{2}$ & 2.16 & 6.60 & 4.67 \\
Capacidad de campo (\%) $^{3}$ & 16.30 & 24.52 & 14.48 \\
Porosidad $\left(\mathrm{m}^{3} / \mathrm{m}^{3}\right)^{3}$ & 0.35 & 0.37 & 0.426 \\
Textura $^{4}$ & Franco & Franco & Franco \\
& limoso & arcilloso & arenoso \\
\hline
\end{tabular}

Métodos analíticos: ${ }^{1}$ ASTM D4972-95a, ${ }^{2}$ ASTM D2974-87, ${ }^{3} \mathrm{~F} 1815-11,{ }^{4} \mathrm{~F} 1632-98 \mathrm{a}$

Respecto a la materia orgánica, el suelo que presentó mayor porcentaje fue el de Yecapixtla con un $6.6 \%$ y una textura franco arcillosa (Cuadro II). Los experimentos de adsorción realizados para cada uno de los suelos indicaron que hubo mayor adsorción de atrazina en este suelo, en comparación con los de Huautla (franco arenoso, con $4.67 \%$ de materia orgánica) y de Moyotepec (franco limoso, con $2.16 \%$ de materia orgánica). Se obtuvieron resultados similares a los realizados por Ling et al. (2005) quienes demostraron que en presencia de materia orgánica y arcilla se favorece el efecto de la adsorción, en tanto que los procesos de biodegradación se limitan. Los valores de adsorción (Cuadro III) indicaron que este proceso fue mayor cuando se aplicó a los suelos atrazina al $100 \%$ en comparación con gesaprim 
CUADRO III. CONSTANTES DE ADSORCIÓN DE FREUNDLICH DE ATRAZINA EN SUELOS AGRÍCOLAS DEL ESTADO DE MORELOS

\begin{tabular}{lrccccccc}
\hline \multirow{2}{*}{ Muestra } & \multicolumn{3}{c}{ Gesaprim } & $(43 \%$ de atrazina $)$ & & \multicolumn{4}{c}{ Atrazina $(100 \%)$} \\
\cline { 2 - 4 } \cline { 7 - 9 } & $\mathrm{Kd}$ & $1 / \mathrm{n}$ & $\mathrm{r}$ & & $\mathrm{Kd}$ & $1 / \mathrm{n}$ & $\mathrm{r}$ \\
\hline Yecapixtla & 12.883 & 0.0120 & 0.874 & & 21.105 & 0.0089 & 0.869 \\
Huautla & 2.676 & 0.0217 & 0.909 & & 1.0198 & 0.0414 & 0.896 \\
Moyotepec & 7.554 & 0.0180 & 0.904 & & 18.414 & 0.0098 & 0.989 \\
\hline
\end{tabular}

$\mathrm{Kd}=$ constantes de adsorción, $\mathrm{n}=$ coeficiente empírico de Freundlich, $\mathrm{r}=$ coeficente de correlación

(43\% atrazina), lo anterior se debe posiblemente a la presencia de agentes surfactantes y humectantes en gesaprim que pueden favorecer que el compuesto activo sea menos hidrófobo. También se puede observar la relación que existe entre el coeficiente de adsorción (Cuadro III) y la capacidad de campo (Cuadro II), donde el suelo arcilloso con valores más altos de capacidad de campo $(24.52 \%)$ presentó un coeficiente de adsorción superior para las dos presentaciones de atrazina aplicadas (12.8830 y $21.1050 \mathrm{~L} / \mathrm{kg}$ gesaprim y atrazina, respectivamente). Mientras que en el suelo franco arenoso con una capacidad de campo inferior $(14.48 \%$ ), el coeficiente de adsorción fue menor (2.6761 y 1.0198 para gesaprim y atrazina, respectivamente). Estos resultados indican que entre menor sea la cantidad de arcilla contenida en el suelo y mayor la cantidad de arena, el coeficiente de adsorción disminuye (Wang y Keller 2009).

Las cinéticas de degradación para los suelos donde se aplicó atrazina $100 \%$ se presentan en la figura 2, en la que se observa que los suelos de Moyotepec y Yecapixtla, presentaron una tendencia exponencial muy similar para ambos suelos con valores del 52.7 $\%$ y $72.9 \%$, respectivamente. Para el suelo de Huautla, durante los primeros ocho días se observó que la degradación fue más rápida, alcanzando finalmente un $65.8 \%$. Por tanto, al observar en los tres suelos la

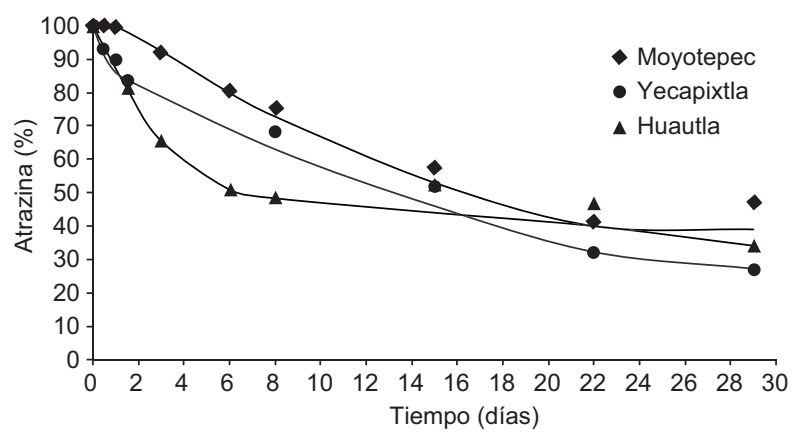

Fig. 2. Cinética de degradación de atrazina $100 \%$, en suelos de tres regiones del estado de Morelos biodegradación del herbicida, los datos sugieren que las muestras de suelo contenían microorganismos capaces de asimilar a la atrazina como fuente de carbono y nitrógeno, aunque no se utilizó un testigo de cada uno de los sistemas o condiciones con suelos estériles para comparar contra suelos con microorganismos. La diferencia de velocidades puede ser influenciada por diversos factores como el $\mathrm{pH}$, la materia orgánica, la textura del suelo o la presencia de nutrientes. Por ejemplo las diversas fuentes de carbono y nitrógeno mineral en el suelo, limitan la biodegradación de la atrazina debido a que los microorganismos utilizan preferentemente fuentes de nutrientes que están disponibles para su metabolismo en lugar de emplear la molécula del herbicida (Guillén-Garcés et al. 2007, Nilanjana et al. 2012). Por tanto, esto se refleja en las diferentes cinéticas de los suelos estudiados y en los parámetros cinéticos obtenidos entre los que están las velocidades de biodegradación, la vida media y los porcentajes de degradación (Cuadro IV), calculados a partir de las ecuaciones 1 y 2 .

Por otro lado, se observó, que en el suelo de Huautla, el herbicida se encontró con mayor biodisponibilidad que en los otros dos suelos, ya que las constantes de adsorción de Freundlich fueron menores $(2.6761$ y $1.0198 \mathrm{~L} / \mathrm{kg}$ para gesaprim y atrazina, respectivamente) que en los suelos de Moyotepec y Yecapixtla (Cuadro III). Lo que puede deberse a que la concentración de materia orgánica en el suelo de Hualtla fue de $4.67 \%$, que es una cantidad intermedia en comparación con los otros dos suelos, además la textura de su suelo se clasifica como franco arenosa, y entre menor presencia de materia orgánica se limita el proceso de adsorción en el mismo (Ling et al. 2005) y se favorece la disponibilidad del herbicida a los microorganismos. Hay que considerar que aún cuando las muestras de los tres suelos se adquirieron de sitios agrícolas donde se aplica frecuentemente el herbicida y por ende, se lograrían favorecer los procesos de inducción de enzimas que catalizan las reacciones de biodegradación, las comunidades 
CUADRO IV. PARÁMETROS CINÉTICOS OBTENIDOS EN LOS EXPERIMENTOS DE DEGRADACIÓN DE ATRAZINA AL 100\%, EN SUELOS AGRÍCOLAS DEL ESTADO DE MORELOS

\begin{tabular}{lcccccc}
\hline Parámetro & Moyotepec & $\mathrm{r}$ & Yecapixtla & $\mathrm{r}$ & Huautla & $\mathrm{r}$ \\
\hline Velocidad de biodegradación (1/d) & 0.0411 & & 0.045 & & 0.1114 & \\
Vidas medias (d) & 17 & 0.9921 & 16 & 0.9893 & 6 & 0.9989 \\
Degradación (\%) & 52.7 & & 72.9 & & 65.8 & \\
\hline
\end{tabular}

$\mathrm{r}=$ coeficente de correlación

CUADRO V. PARÁMETROS CINÉTICOS OBTENIDOS EN LOS EXPERIMENTOS DE DEGRADACIÓN DE GESAPRIM (43 \% ATRAZINA), EN SUELOS AGRÍCOLAS DEL ESTADO DE MORELOS

\begin{tabular}{lcccccc}
\hline Parámetro cinético & Moyotepec & $\mathrm{r}$ & Yecapixtla & $\mathrm{r}$ & Huautla & $\mathrm{r}$ \\
\hline Velocidad de degradación (1/d) & 0.0055 & & 0.0316 & & 0.0174 & \\
Vidas medias (d) & 126 & 0.9971 & 18 & 0.9189 & 40 & 0.9925 \\
Degradación (\%) & 21.4 & & 71.8 & & 50.1 & \\
\hline
\end{tabular}

$\mathrm{r}=$ coeficente de correlación

microbianas presentes en las muestras podrían ser diferentes y entonces sería otra causa de las diferentes velocidades de biodegradación entre los suelos.

$\mathrm{Al}$ hacer la comparación entre las cinéticas con atrazina al $100 \%$ y gesaprim (atrazina al $43 \%$ ) se observó que en el caso del suelo de Yecapixtla las velocidades de biodegradación fueron de $0.0451 / \mathrm{d}$ para atrazina $100 \%$ y $0.03161 / \mathrm{d}$ para gesaprim ( $43 \%$ atrazina), con vidas medias de 16 y 18 días, respectivamente. Como se puede observar no hay influencia por la presencia de los surfactantes y emulsificantes contenidos en el gesaprim ya que sólo difiere la vida media de la atrazina por dos días. En comparación con los suelos de Huautla y Moyotepec (Cuadro IV y V) en los que la diferencia entre las vidas medias fue de casi de un orden de magnitud entre los resultados obtenidos para atrazina al $100 \%$ y el gesaprim (atrazina al $43 \%$ ), la explicación podría deberse a que la concentración de arcilla y de materia orgánica contenida en el suelo de Yecapixtla fue mayor (6.60\%), lo que hubiera limitado el efecto de la presencia de los surfactantes y humectantes sobre la biodegradación del herbicida. En los suelos donde la cantidad de arcillas y materia orgánica fueron menores (Moyotepec y Huautla con 2.16 y $4.67 \%$ de materia orgánica, respectivamente) las cinéticas de biodegradación con gesaprim (43\% atrazina) fueron más lentas en comparación con los experimentos realizados con atrazina $100 \%$ (Fig. 3). Lo anterior puede ser debido a que cuando la presencia de arcillas y materia orgánica son menores, los surfactantes y humectantes contenidos en el gesaprim ( $43 \%$ atrazina) aumentan la disponibilidad de compuestos que compiten como nutriente para los microorganismos. Mientras que con alto contenido de arcilla y materia orgánica como los suelos de Yecapixtla con $6.60 \%$ de materia orgánica, los procesos de adsorción tienen mayor influencia aún en presencia de los componentes contenidos en el gesaprim.

Respecto a la textura de suelo y su relación con las velocidades de biodegradación de atrazina al $100 \%$, el suelo con textura limosa y arcillosa (Moyotepec y Yecapixtla) puede tener mayor riesgo de infiltración si el único proceso de disipación fuera la biodegradación, lo anterior debido a que fueron las velocidades más lentas. En relación con el gesaprim (43\% atrazina), el mayor riesgo de infiltración es en los suelos de Moyotepec y Huautla debido a que la biodegradación fue más lenta, lo que aumentaría el riesgo de infiltración cuando se aplica en esta presentación.

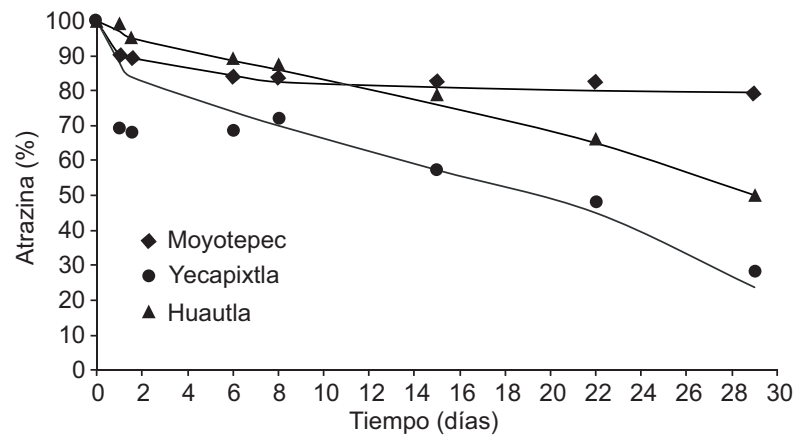

Fig. 3. Cinética de degradación de gesaprim (43\% atrazina), de tres regiones del estado de Morelos 


\section{CONCLUSIONES}

Los experimentos de biodegradación permitieron obtener los parámetros cinéticos de atrazina al $100 \%$ y gesaprim (43\% atrazina) en diversas muestras de suelo agrícola como franco limoso (Moyotepec), franco arcilloso (Yecapixtla) y franco arenoso (Huautla), con diferentes cantidades de materia orgánica $(2.16,4.67$ y $6.60 \%$, respectivamente).

La presencia de emulsificantes y surfactantes limitan el proceso de biodegradación de atrazina en suelos franco limoso (Moyotepec) y franco arenoso (Huautla), debido probablemente a que no favorecen la disponibilidad de diversos compuestos en el suelo, los cuales pueden servir como nutrientes para los microorganismos.

En suelos con alto contenido de arcilla (Yecapixtla) y materia orgánica (Huautla), la presencia de aditivos (surfactantes y humectantes) en gesaprim (atrazina $43 \%$ ) no influye sobre la biodegradación del herbicida, aunque es necesario hacer estudios sobre la influencia de diferentes concentraciones de surfactantes y emulsificantes en los procesos de disipación de los herbicidas.

\section{REFERENCIAS}

ASTM (1987). D2974-87. Standard test methods for moisture, ash, and organic matter of peat and other organic soils. American Society for Testing and Materials. ASTM International. 29 de mayo de 1987.

ASTM (1995). D4972-95a. Standard test method for $\mathrm{pH}$ of soils. American Society for Testing and Materials. ASTM International. 15 de julio de 1995.

ASTM. (1998). F1632-98a. Standard test methods for particle size analysis and sand shape grading of golf course putting green and sports field rootzone mixes. American Society for Testing and Materials. ASTM International. 10 de noviembre de 1998.

ASTM (2011). F1815-11. Standard test method for saturated hydraulic conductivity, water retention, porosity, particle density, and bulk density of putting green and sports turf root zones. American Society for Testing and Materials. ASTM International. 1 de abril de 2011.

ATSDR (2003). CAS\#: 1912-24-9. Resumen de Salud Pública (atrazina). Agencia para Sustancias Tóxicas y Registro de Enfermedades. Departamento de Salud y Servicios Humanos de EUA. Resumen. Atlanta, EUA, 7 pp.

Ahmad R. y Rahman A. (2009). Sorption characteristics of atrazine and imazethapyr in soils of New Zealand:
Importance of independently determined sorption data. J. Agric. Food Chem. 57, 10866-10875.

DOI: $10.1021 / \mathrm{jf} 901365 \mathrm{j}$

Beth J. y Colangelo A. (2006). European Union bans atrazine, while the United States negotiates continued use. Int. J. Occup. Environ. Health. 12, 260-267.

DOI: 10.1179/oeh.2006.12.3.260

Buttiglieri G, Migliorisi L. y Malpei F. (2011). Adsorption and removal at low atrazine concentration in an MBR pilot plant. Water Sci. Technol. 63, 1334-1340.

DOI: $10.2166 /$ wst.2011.130

CICLOPLAFEST (2004). Catálogo de plaguicidas de la Comisión Intersecretarial para el control del proceso y uso de plaguicidas, fertilizantes y sustancias tóxicas. CICLOPLAFEST. México D.F., México, 493 pp.

Cilia L., Lozano de Yunda A., Guerrero-Dallos J. A., Pérez L. E., Olarte I. y Acevedo B. (2003). Comportamiento y destino ambiental de la atrazina en el suelo: Detección por HPLC y ${ }^{14} \mathrm{C}$ de las concentraciones ambientales bajo condiciones controladas y de campo en SaldañaTolima. Agronomía Colombiana 21, 29-48.

FAO (2000). APÉNDICE 1. Forma de evaluar la contaminación del suelo sobre el terreno. Food and Agriculture Organization. Formato de captura de información [en línea]. http://www.fao.org/docrep/005/x2570s/ X2570S05.htm\#app1.1 22/02/2015

FAO (2003). Métodos sencillos para acuicultura, suelo. Food and Agriculture Organization. Manual de referencia [en línea]. ftp://ftp.fao.org/fi/cdrom/fao_training/ FAO_Training/General/x6706s/Index.htm 11/04/2015

FAO (2005). Proceedings of the Asia regional workshop on the implementation, monitoring and observance of the international code of conduct on the distribution and use of pesticides. Memorias en Congreso. Food and Agriculture Organization. Bangkok, Tailandia, 249 pp.

Farré M., Abanades S., Peiró A. M., Torrens M., O’Mathúna B., Segura M. y de la Torre R. (2007). Pharmacological interaction between 3, 4-methylenedioxymethamphetamine (ecstasy) and paroxetine: pharmacological effects and pharmacokinetics. J. Pharmacol. Exp. Ther. 32, 954-962.

DOI: $10.1124 /$ jpet.107.129056

Fournier D., Halasz A., Spain J., Fiurasek P. y Hawari J. (2002). Determination of key metabolites during biodegradation of hexahidro 1, 3, 5 Trinitro 1, 3, 5 Triazine with Rhodococcussp Strain DN 22. Appl. Environ. Microbiol. 68, 166-172.

Guillén-Garcés R. A., Hansen A. M. y Afferden van M. (2007). Mineralization of atrazine in agricultural soil: Inhibition by nitrogen. Environ. Toxicol. Chem. 26, 844-850. DOI: 10.1897/06-328R.1

Golla V., Nelms J., Taylor R. y Mishra S. (2011). Pesticide concentrations in drinking water from farm homes: 
Variation between community water supply and wellwater. J. Environ. Sci. Eng. 5, 955-961.

González-Márquez L.C. y Hansen A.M. (2009). Adsorción y mineralización de atrazina y relación con parámetros de suelo del DR 063 Guasave, Sinaloa. Rev. Mex. Cienc. Geol. 26, 587-599.

Hansen A.M., Van Afferden M., Quintero O., Martínez E. y Guillén R.A. (2001). Migración de agroquímicos en suelo agrícola y cuantificación en el agua drenada a nivel parcelario (Etapa III), Informe Técnico, Proyecto TH-2015, Instituto Mexicano de Tecnología del Agua y Comisión Nacional del Agua, Morelos, México, 80 pp.

Hamaker J. W. (1972). Descomposition: quantitative aspects. En: Organic chemical in the soil environment Vol. 1. (C. A. I. Goring y J. W. Hamaker, Eds.). Dekker, Nueva York, EUA, pp. 253-340.

Hayes T. B., Collins A., Lee M., Mendoza M., Noriega N., Stuart A. A y Vonk A. (2002). Hermaphroditic, demasculinized frogs after exposure to the herbicide atrazine at low ecologically relevant doses. Proc. Natl. Acad. Sci. USA 99, 5476-5480.

DOI: $10.1073 /$ pnas. 082121499

Huang G., Li Q. y Zhang X. (2003). Adsorption and desorption of atrazine by three soils. Bull. Environ. Contam. Toxicol. 71, 655-661.

DOI: $10.1007 / \mathrm{s} 00128-003-0183-1$

INEGI (2005). Anuario Estadístico de Morelos. Instituto Nacional de Estadística y Geografía [en línea]. http:// www.inegi.org.mx/est/contenidos/espanol/sistemas/ aee05/info/mor/mapas.pdf 05/02/2015

Koskinen W.C. y Clay S. A. (1997). Factors affecting atrazine fate in North Central U.S. Soils. Rev. Environ. Contam. Toxicol. 151, 117-165.

DOI: 10.1007/978-1-4612-1958-3_4

Krutz L. y Shaner D. (2008). Atrazine dissipation in striazine-adapted and nonadapted soil from Colorado and Mississippi: Implications of enhanced degradation on atrazine fate and transport parameter. J. Environ. Qual. 37, 848-857. DOI: 10.2134/jeq2007.0448

Ling W. T., Wang H. Z., Xu J.M. y Gao Y.Z. (2005). Sorption of dissolved organic matter and its effects on the atrazine sorption on soils. J. of Env. Sci. 17, 478-482.

Mahía J. y Díaz-Raviña M. (2007). Atrazine degradation and residues distribution in two acid soils from temperate humid zone. J. Environ. Qual. 36, 826-831. DOI: $10.2134 /$ jeq2006.0477

Ma L. y Selim H.M. (1996). Atrazine retention and transport in soils. Rev. Env. Cont. Tox. 145, 129-73. DOI: $10.1007 / 978-1-4612-2354-22$

Nilanjana D., Agitail E. y Lakshmi V. (2012). Biodegradation of atrazine by Cryptococcus leurentii isolated from contaminated agriculture soil. J. Microbiol. Biotech. Res. 2, 450-457.
OECD (2000). Test No. 106. Adsorption-desorption using a batch equilibrium method. OECD Guidelines for the Testing of Chemicals. Guía. Organization for Economic Cooperation and Development. París, Francia, 43 pp. DOI: 10.1787/9789264069602-en

PMRA (2015). Special review of atrazine: Proposed decision for consultation. Pest Management Regulatory Agency, Health Canada [en línea]. http://www.hc-sc. gc.ca/cps-spc/pest/part/consultations/_rev2015-11/ rev2015-11-eng.php 25/12/2015

Rosen M. J. (1989). Surfactant and interfacial phenomena. 2nd ed. Wiley and Sons, Nueva York, EUA, $616 \mathrm{pp}$.

Ross M., Goberna M., Moreno J. L., Hernández T., García C. e Insam H. (2006). Molecular and physiological bacterial diversity of a semiarid soil contaminated with different levels of formulated atrazine. Appl. Soil Ecol. 34, 93-102. DOI: 10.1016/j.apsoil.2006.03.010

Rusiecki J. A., De Roos A, Lee W. J., Dosemeci M., Lubin J. H., Hoppin J. A., Blair A. y Alavanja M. C. (2004). Cancer incidence among pesticide applicators exposed to atrazine in the agricultural health study. J. Natl. Cancer Inst. 96,1375-1382. DOI: 10.1093/jnci/djh264

SAGARPA (2007). Anteproyecto de modificación a la Norma Oficial Mexicana NOM-032-FITO-1995. Requisitos y especificaciones fitosanitarios para la realización de estudios de efectividad biológica de plaguicidas agrícolas y su dictamen técnico. Secretaría de Agricultura, Ganadería y Desarrollo Rural, Pesca y Alimentación. Diario Oficial de la Federación. 11 de agosto de 2015.

SEDESU (2013). Programa de ordenamiento ecológico regional del estado de Morelos. Secretaría de Desarrollo Sustentable [en línea] http://obum.zmcuernavaca. morelos.gob.mx/MORELOS/OET/POEREM_PROPUESTA.pdf 05/02/2015

Schoen S. R. y Winterlin W. R. (1987). The effect of various soil factors and amendments on the degradation of pesticide mixtures. J. Environ. Sci. Health, Part B. 22, 347-377. DOI: 10.1080/03601238709372561

Singh A. K. y Cameotra S. S. (2014). Influence of microbial and synthetic surfactant on the biodegradation of atrazine. Environ. Sci. Pollut. Res. 21, 2088-2097. DOI: $10.1007 / \mathrm{s} 11356-013-2127-6$

Tyess D.L., Shea P.J. y Parkhurst A.M. (2006). Mineralization potential of atrazine and degradation intermediates from clustered characteristics in inoculated soils. Soil Sediment Contam. 15, 87-102. DOI: $10.1080 / 15320380500364440$

UNEP (2002). Central America and the caribbean regional report, regionally based assessment of persistent toxic substances. United Nations Environment Programme. Ginebra, Suiza, 145 pp. 
Villada-Canela M. (2006). Estimación del riesgo de contaminar mantos freáticos por infiltración de herbicida Atrazina en distritos de riego en México. Tesis de Maestría. Facultad de Ingeniería, Universidad Nacional Autónoma de México. Jiutepec, Morelos, México, $128 \mathrm{pp}$.

Wang X., Guo X., Yang Y., Tao S. y Xing B. (2011). Sorption mechanisms of phenanthrene, lindane, and atrazine with various humic acid fractions from a single soil sample. Environ. Sci. Technol. 45, 2124-2130.

DOI: $10.1021 /$ es $102468 \mathrm{z}$

Wang P. y Keller A. A. (2009). Sorption and desorption of atrazine and diuron onto water dispersible soil primary size fractions. Water Res. 43, 1448-1456.

DOI: 10.1016/j.watres.2008.12.031
Ware G. W., y Whitacre D. M. (2004). The pesticide book. 6th ed. Meister Publishing Company. Willoughby, Ohio, EUA, 488 pp.

Weber J. B. (1995). Physicochemical and mobility studies with pesticides. En: Agrochemical environmental fate state of the art (M. L. Leng, E. M. K. Leovey y P. L. Zubkoff, Eds.). CRC Press, Inc. Washington, EUA, pp. 99-105.

Worthing C. R. y Walker S. B. (1987). Atrazine. En: The pesticide manual-A world compendium. (C.R. Worthing y S.B. Walker, Eds.) Thornton Heath, Londres, Reino Unido, pp. 36-37. 